\title{
Caracterización Fisicoquímica de Pan con Sustitución Parcial de Harina de Trigo por Harina de Quinua (chenopodium quinoa willd) y Kiwicha (amaranthus caudatus I.) Germinadas Physicochemical Characterization of Bread with Partial Sustitución of wheat flour for Quinoa flour (Chenopodium quinoa willd) and Kiwicha (Amaranthus caudatus I.) Germinated
}

\author{
Dr. Jorge Wilmer Elías Silupu ${ }^{\text {; }}$; jelias s@hotmail.es \\ ORCID: https://orcid.org/0000-0002-0857-4258 \\ Universidad Nacional Ciro Alegría \\ Dra. Cecilia Edith García Rivas Plata ${ }^{b}$ Cegrivas@gmail.com \\ ORCID: https://orcid.org/0000-0001-7254-1162 \\ Universidad Nacional Ciro Alegria \\ Mg. Ronald Pérez Salcedo ${ }^{a}$; celyauris13@gmail.com \\ ORCID: https://orcid.org/0000-0001-9251-1788 \\ Universidad Nacional José María Arguedas \\ Mg. Celia Rocio Yauris Silvera ${ }^{a}$; ronaldps 13@gmail.com \\ ORCID: https://orcid.org/0000-0003-2681-7724 \\ Universidad Nacional José María Arguedas
}

Recibido: Abril/ 10/2021-Revisado: Mayo / 15 /2021- Publicado: Junio /30/2021

\section{RESUMEN}

En esta investigación se realizó pan con sustitución parcial de harina de trigo (65\%) por harina de quinua germinada (20, 15 y 10\%) y harina de kiwicha germinada (15, 20 y 25\%) para caracterizar fisicoquímicamente realizando análisis de peso, textura, volumen específico, porosidad y composición proximal. Asimismo, para el análisis estadístico se utilizó el diseño de mezclas empleando el estadístico Statgraphics Centurión XVI y Excel 2016. Por otra parte, la caracterización de las propiedades físicas del pan se determinó mediante los siguientes métodos: para el peso (método de Cauvain S. y Young L. (1998)); textura (método $N^{\circ} 74-10 A$ de la AACC (2000)); volumen especifico (modificación del método $N^{\circ} 10-05$ de la AACC (2000)); porosidad (método óptico (Sahin, G., 2009; Karathanos y Saravacos,1992)). En consecuencia, para la caracterización de las propiedades químicas del pan, se utilizó los siguientes métodos: humedad (NTP 209.264:2013); proteína (NTP 209.262:2013); grasa (NTP 209.263:2013); cenizas (NTP 209.265:2013); fibra (FAO 14/7); el contenido de carbohidratos se determinó por diferencia de peso. Entonces el tratamiento más adecuado para el pan con un nivel de sustitución de 10\% de harina de quinua germinada (HQG), 25\% de harina de kiwicha germinada (HKG) y 65\% de harina de trigo (HT), el resultado del análisis físico como el peso, textura y porosidad de los panes con sustitución fue ascendente, en tanto a los valores del volumen específico disminuyeron; en el aspecto proximal, este tratamiento presento un \% alto de proteínas (8.64\%), humedad (17.78\%), grasa (11.39\%), ceniza (1.49\%), fibra (1.72\%) y carbohidratos (59.02\%) respectivamente. Al observar estos resultados, se puede indicar que se ha incrementado el contenido proteico, que es muy importante en la alimentación.

Palabras claves: Germinado, Quinua, Kiwicha, Pan, Sustitución y Caracterización. 


\begin{abstract}
In this research, bread was made with partial replacement of wheat flour (65\%) by sprouted quinoa flour (20, 15 and 10\%) and sprouted kiwicha flour (15, 20 and 25\%) to characterize physicochemically by performing weight analysis, texture, specific volume, porosity and proximal composition. Likewise, for the statistical analysis, the mixture design was used using the Statgraphics Centurion XVI and Excel 2016 statistics. On the other hand, the characterization of the physical properties of the bread was determined by the following methods: for the weight (method of Cauvain S. and Young L. (1998)); textura (AACC Method No. 74-10A (2000)); specific volume (modification of method No. 10-05 of the AACC (2000)); porosity (optical method (Sahin, G., 2009; Karathanos and Saravacos, 1992)). Consequently, for the characterization of the chemical properties of bread, the following methods were used: humidity (NTP 209.264: 2013); protein (NTP 209.262: 2013); fat (NTP 209.263: 2013); ashes (NTP 209.265: 2013); fiber (FAO 14/7); carbohydrate content was determined by weight difference. So the most suitable treatment for bread with a substitution level of $10 \%$ sprouted quinoa flour (HQG), 25\% sprouted kiwicha flour (HKG) and 65\% wheat flour (HT), the result of the analysis physical as the weight, texture and porosity of the loaves with substitution was ascending, while the values of the specific volume decreased; In the proximal aspect, this treatment presented a high\% of proteins (8.64\%), humidity (17.78\%), fat (11.39\%), ash (1.49\%), fiber $(1.72 \%)$ and carbohydrates (59.02\%) respectively. By observing these results, it can be indicated that the protein content, which is very important in the diet, has increased.
\end{abstract}

Keywords: Sprouts, Quinua, Kiwicha, Bread, Substitution and Characterization.

\title{
Introducción
}

El pan es un producto altamente consumido por la población, en este sentido es importante ofrecer nuevas variedades de harinas de diversos tipos de cereales y granos andinos, el cual aporta un alto valor nutricional al producto.

La harina de trigo, es el ingrediente principal para la elaboración del pan, pero es deficiente en lisina (Edel y Rosell, 2007). Para contrarrestar la deficiencia de aminoácidos en el pan, se puede realizar el enriquecimiento de harinas de cereales ricas en lisina procedentes de los granos de quinua y kiwicha, cuya calidad proteica (FAO/ OMS/ ONU, 1985).

La gran mayoría de investigaciones, se centraron en estudiar el efecto de la sustitución de la harina de trigo por harina obtenida a partir de distintos granos, la 
interacción de ciertos parámetros de producción del pan que presenta sustitución con respecto a sus características fisicoquímicas, microbiológicas, organolépticas y tiempo de vida útil, parámetros como el tiempo y temperatura de fermentación y horneado, entre otros, no se encontró investigación alguna que muestre la interacción del pan con sustitución parcial de harina de trigo por harina de quinua germinada y harina de kiwicha germinada en algunas características fisicoquímicas del pan.

Por lo que la presente investigación tiene como objetivo general:

Caracterizar las propiedades fisicoquímicas del pan con sustitución parcial de harina de trigo por harina de quinua (Chenopodium quinoa willd) y kiwicha (Amaranthus caudatus I.) germinadas.

Y como objetivos específicos:

Elaborar pan con sustitución parcial de harina de trigo por harina de quinua (Chenopodium quino willd) y kiwicha (Amaranthus caudatus I.) germinadas.

Caracterizar las propiedades físicas (peso, textura, volumen específico y porosidad) del pan con sustitución parcial de harina de trigo por harina de quinua (Chenopodium quino willd) y kiwicha (Amaranthus caudatus I.) germinadas.

Caracterizar las propiedades químicas (\% humedad, \% proteína, \% ceniza, $\%$ grasa, \% fibra y \% carbohidrato) del pan con sustitución parcial de harina de trigo por harina de quinua (Chenopodium quino willd) y kiwicha (Amaranthus caudatus I.) germinadas.

\section{Materiales y Métodos}

Reactivos:

- Éter de petróleo

- Hidróxido de sodio al $1.25 \%$

\section{Materia prima:}


- Harina de trigo

- Harina de quinua germinada

- Harina de kiwicha germinada

\section{Equipos}

- Horno

- Cámara de fermentación

- Amasadora

- Mezcladora

- Divisora

- Balanza digital

- Estufa

- Texturómetro

- Campana extractora de gas

\section{Procesos para la germinación de los granos de quinua y kiwicha}

El método utilizado para la germinación de la quinua y kiwicha fue el método diferente o casos especiales establecida por Botero (2011) que se muestra en la figura 1.

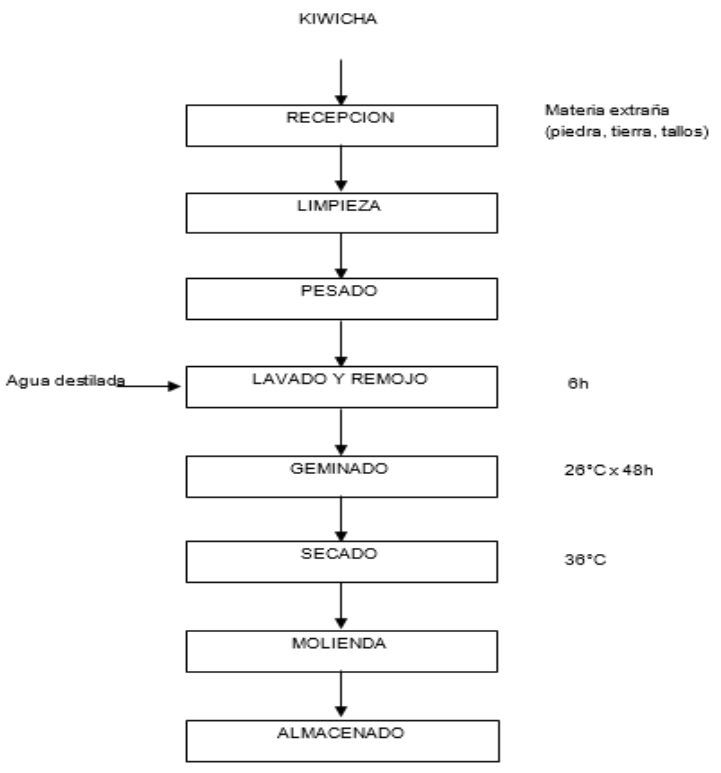


Figura 1. Diagrama para la obtención de harina de quinua y kiwicha germinada.

Proceso para la obtención de pan con sustitución parcial de harina geminada de quinua (Chenopodium quinoa) y kiwicha (Amaranthus caudatus $I_{\text {.). }}$.

Las mezclas de harina se realizaron por peso en base seca, las proporciones de harina de quinua germinada (HQG)/harina de Kiwicha germinada (HKG)/ harina de trigo $(\mathrm{HT})$ tal como se muestra en la tabla 1 y son las siguientes: T1 $(20 ; 15 ; 65)$, T2 $(15 ; 20 ; 65)$ у T3 $(10 ; 25 ; 65)$.

Tabla 1: Porcentaje de sustitución de harina de trigo por harina germinada de quinua y kiwicha.

\begin{tabular}{lll}
\hline $\begin{array}{c}\text { Harina de quinua } \\
\text { germinada } \%\end{array}$ & \multicolumn{1}{c}{$\begin{array}{c}\text { Harina de } \\
\text { Kiwicha } \\
\text { germinada } \%\end{array}$} & $\begin{array}{c}\text { Harina de trigo } \\
\%\end{array}$ \\
\hline 20.0 & 15.0 & 65.0 \\
10.0 & 25.0 & 65.0 \\
10.0 & 15.0 & 75.0 \\
15.0 & 20.0 & 65.0 \\
15.0 & 15.0 & 70.0 \\
10.0 & 20.0 & 70.0 \\
13.3333 & 18.3333 & 68.3333 \\
16.6667 & 16.6667 & 66.6667 \\
11.6667 & 21.6667 & 66.6667 \\
16.6667 & 16.6667 & 71.6667 \\
\hline
\end{tabular}


En la Figura 2 se presenta el diagrama para la elaboracion de pan con sustitucion por harina de quinua y kiwicha germinada.

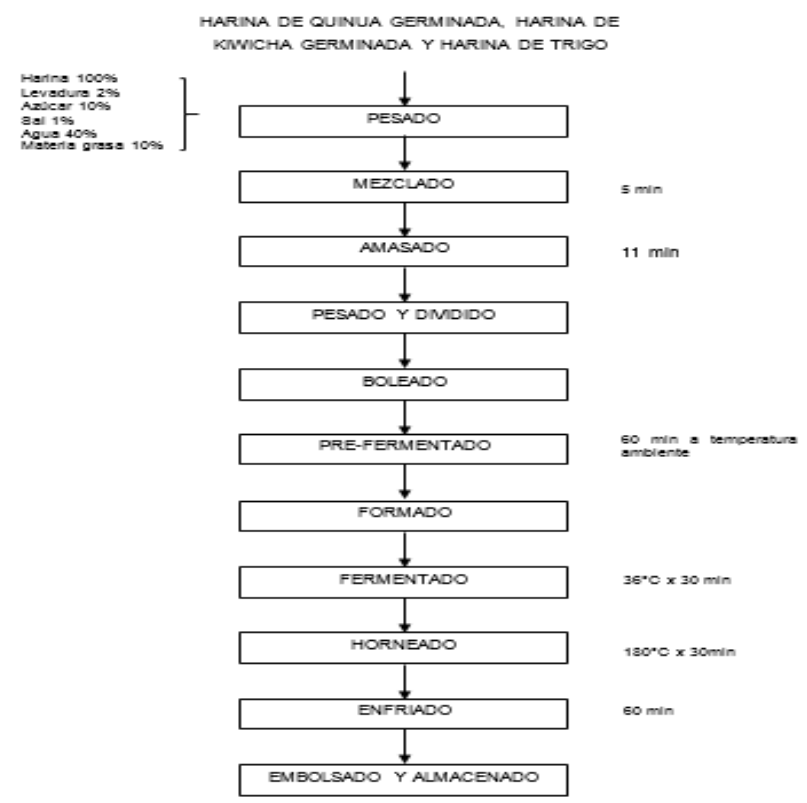

Figura 2. Diagrama de flujo para la elaboración del pan

Análisis físico del pan. Los análisis físicos fueron los siguientes:

Peso: Se realizó con una balanza analítica.

$>$ Textura: Se determinó mediante el método 74-10A de la AACC (2000).

> Volumen específico: La medición del volumen específico se llevó a cabo usando una modificación del método 10-05 de la AACC (2000). Finalmente se aplicó la siguiente ecuación:

$$
V=\pi \times r^{2} \times D \ldots \ldots . E \boldsymbol{E} \text {. (1) }
$$

Donde:

$\mathrm{V}=$ volumen del pan $(\mathrm{cm})^{3}$

$\mathrm{r}=$ radio del cilindro $(\mathrm{cm})$

$\mathrm{D}=$ distancia desplazamiento desde la marca $(\mathrm{cm})$

Análisis químico del pan. Se realizó los siguientes análisis:

Determinación de la humedad: Se determinó mediante la Norma Técnica Peruana NTP 209.264:2013. 
> Determinación de proteína: Se determinó mediante la Norma Técnica Peruana NTP 209.262:2013.

> Determinación de grasa: Se determinó mediante la Norma Técnica Peruana NTP 209.263:2013.

> Determinación de cenizas: Se determinó mediante la Norma Técnica Peruana NTP 209.265:2013.

> Determinación de fibra: Se determinó de acuerdo Food and agriculture organization FAO 14/7, por hidrolisis ácido alcalino.

Determinación de carbohidratos: Se determinó por diferencia de peso, esto se realizó después de completar los análisis de cenizas, fibra, grasa, proteínas y humedad, todo en base seca.

\section{Resultados y Discusión}

Caracterización de las propiedades físicas del pan: Las propiedades físicas evaluadas del pan con sustitución parcial de harina de trigo por harina de quinua y kiwicha germinadas fueron: peso, textura, volumen especifico y porosidad.

Peso final del pan: En la tabla 2 se muestra los resultados del peso final (g) de las tres formulaciones del pan de las cuales sacando un promedio por cada tratamiento se puede apreciar que el menor peso se encuentra en el T3 con 59g.

\begin{tabular}{lllll}
$\begin{array}{c}\text { Tabla 2 } \\
\text { Tratamiento }\end{array}$ & Resultados del peso de acuerdo a los tratamientos \\
\hline & & $\begin{array}{l}\text { Peso inicial } \\
\text { (g) }\end{array}$ & Peso final (g) & Peso perdido (g) \\
$\mathbf{T}_{1}$ & $\mathbf{r}_{1.1}$ & 70 & 60 & 10 \\
& $\mathbf{r}_{1.2}$ & 70 & 60 & 10 \\
& $\mathbf{r}_{1.3}$ & 70 & 61 & 9 \\
$\mathbf{T}_{2}$ & $\mathbf{r}_{2.1}$ & 70 & 61 & 9 \\
& $\mathbf{r}_{2.2}$ & 70 & 60 & 10 \\
& $\mathbf{r}_{2.3}$ & 70 & 61 & 9 \\
$\mathbf{T}_{3}$ & $\mathbf{r}_{3.1}$ & 70 & 60 & 10 \\
& $\mathbf{r}_{3.2}$ & 70 & 60 & 10 \\
\hline
\end{tabular}


Y en la figura 3 se puede observar la codificación del rango de los valores predichos para el peso del pan en colores, extendiéndose desde el azul en 59.0 hasta el rojo en 61. En la zona roja es donde se encuentra el mayor valor de peso que se obtuvo, en esta región se encuentra el óptimo peso las cuales corresponden al T1 y al T2.

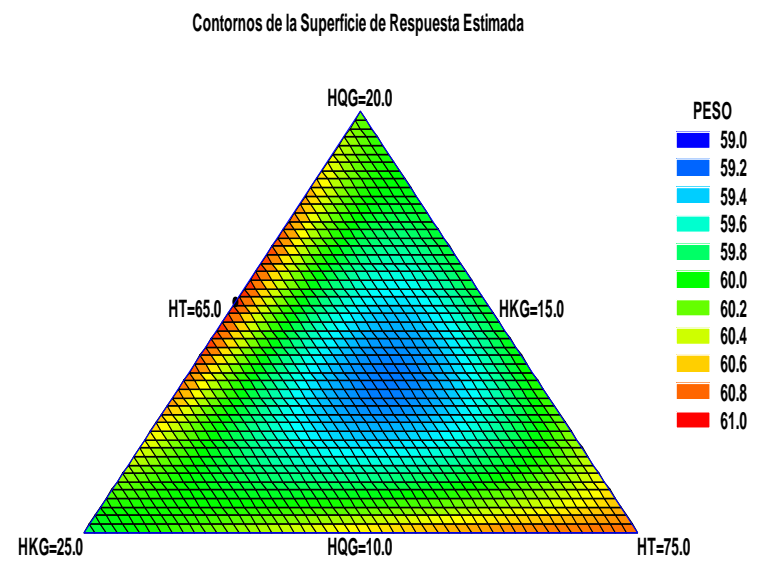

Figura 3. Superficie de respuesta para el peso del pan

Según Arone (2015) a medida que se incrementa la cantidad de harina de trigo existe una mayor pérdida de peso, por lo que este autor sugiere que se incremente las harinas de sustitución, las cuales conllevaran a un incremento de peso significativo; sin embargo, en esta investigación se puede observar que el peso del pan no presento una gradual diferencia debido a que en los tratamientos ya establecidos solo el porcentaje de concentración de harina de quinua germinada y el porcentaje de harina de kiwicha germinada varia, otro causante de que el peso del pan no presente variaciones, se debería a que la formulación del pan se aplicó en función del total de la harina, el peso promedio del pan fue de $60.22 \mathrm{~g}$. 
Textura del pan: En la figura 4 se muestra la textura óptima es 6.15 con una mezcla de $\mathrm{HQG}=10.0 \%$, $\mathrm{HKG}=15.0 \%$ y $\mathrm{HT}=75.0 \%$.

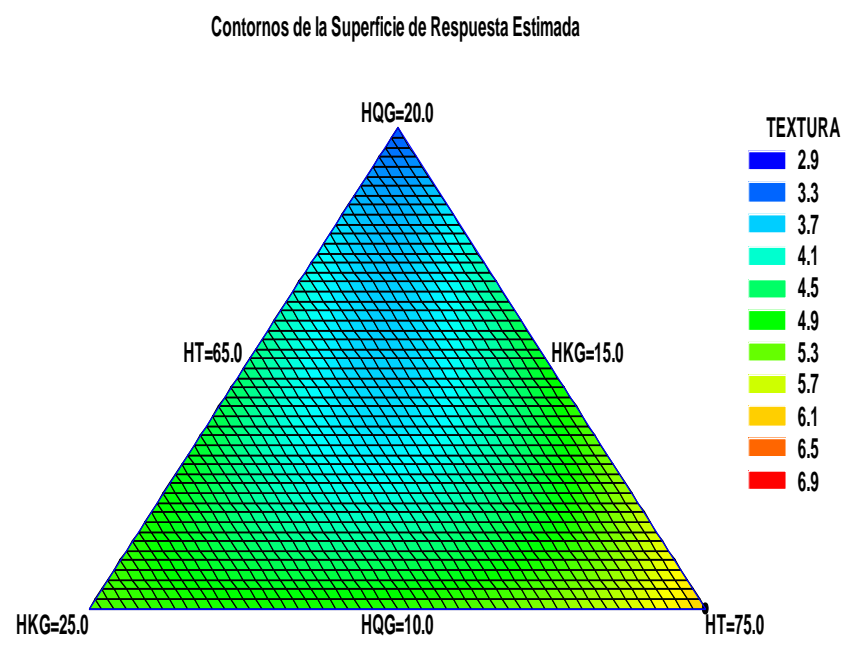

Figura 4. Superficie de respuesta para la textura del pan.

Según las investigaciones realizadas por Vásquez, et al. (2016) registró diferencias significativas las cuales al incrementar la sustitución observó como la textura del pan disminuye considerablemente. Los resultados obtenidos concuerdan con dicho investigador, ya que la textura del pan si presento variaciones significativas esto se debe a medida que disminuye el \% de harina de quinua germinada (HQG) y aumente el \% de harina de kiwicha (HKG), el valor de la textura del pan se incrementa. 
Volumen específico del pan: En la figura 5 se muestra el valor máximo del volumen específico del pan que se alcanza con la HKG, mientras que el valor mínimo se logra con HT, en la zona roja es donde se encuentra el mayor valor de peso que se ha podido obtener. Pero el volumen especifico optimo se encuentra se encuentra en el T1.

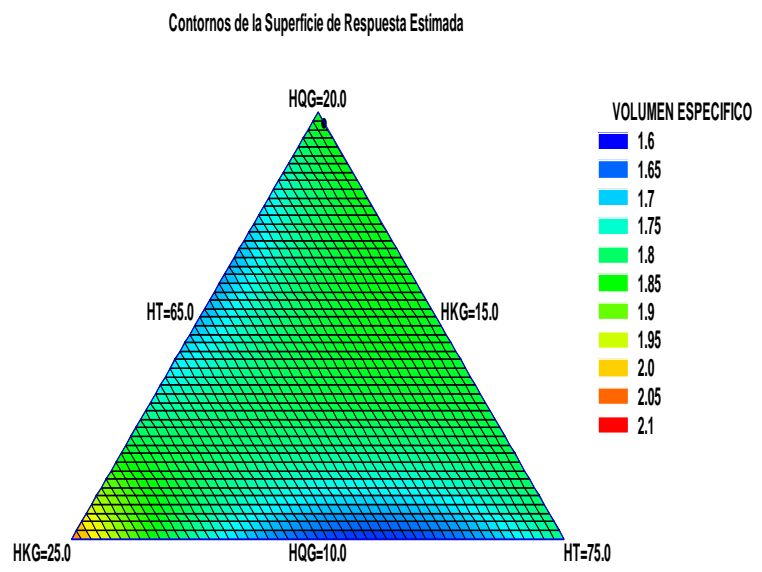

Figura 5. Superficie de respuesta para el volumen especifico del pan.

Según Callejo (2002) menciona que la variación del volumen específico del pan se debe al incremento de proteínas en la masa, además el nivel de sustitución influirá directamente en algunas características físicas del pan tales como el peso, volumen específico y textura. Los resultados obtenidos no concuerdan con lo mencionado por Callejo (2002) el volumen especifico del pan no presentó una diferencia significativa entre los tres tratamientos ya que, del $100 \%$ el $35 \%$ es sustitución de HQG y HKG, pero si lo comparamos con el pan hecho solo con harina de trigo pues existe una variación significativa porque presenta una buena cantidad de gluten.

Porosidad del pan: En la figura 6 se muestra el mayor valor de la porosidad que se ha podido obtener siendo el valor óptimo de $67.9117 \%$ de porosidad con una mezcla óptima de $\mathrm{KQG}=13.1 \%$, $\mathrm{HKG}=18.3 \%$ y $\mathrm{HT}=68.6 \%$. 


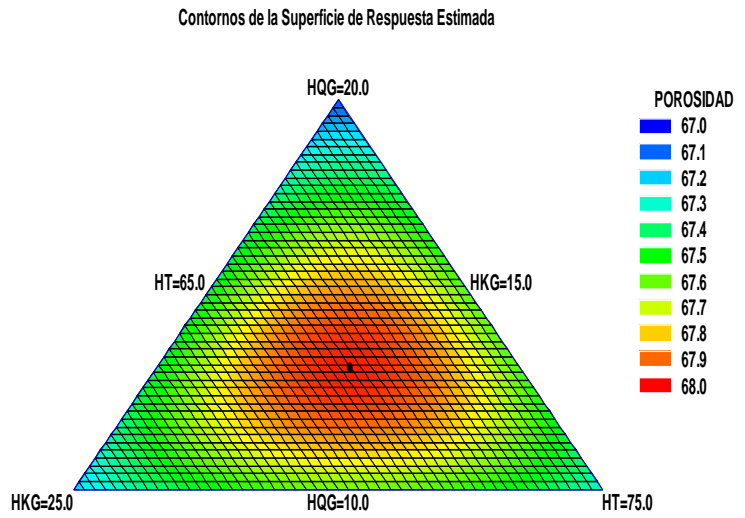

Figura 6. Superficie de respuesta para la porosidad del pan.

Según Tejero (como cito Arone, 2013) menciona que durante la formulación se producen pequeñas burbujas de aire que retiene el $\mathrm{CO}_{2}$ estas celdas, cuando se realiza la sustitución las celdas formadas no soportan la presión del $\mathrm{CO}_{2}$ debido a que existen disminución del gluten el cual es encargado de la elasticidad de la masa, dando como resultado el incremento de alveolos o celdas. La porosidad es una propiedad física que caracteriza la textura, la calidad de los panes y humedad intermedia. Por otro lado, en esta investigación, la porosidad no presenta una diferencia significativa de las cuales el porcentaje de variación en cada tratamiento era el $35 \%$, por otro lado, el $65 \%$ estaba conformado por $\mathrm{HT}$, la porosidad promedio del pan es $67.48 \%$.

Características químicas del pan: Se realizó los análisis:

Humedad del pan: En la figura 7 se observa el valor óptimo para el porcentaje de humedad del pan es 19.24 con una mezcla óptima de $K Q G=13.0 \%$, $H K G=15.0 \%$ y $H T=71.97 \%$. Además, se puede ver que el valor máximo de la humedad del pan se alcanza con la HT, mientras que el valor mínimo se logra con HKG.

Figura 7. Gráfica de trazas para la humedad del pan. 
Según MINSA (2010) menciona que los límites máximos permisibles de humedad en la industria panadera es de 23\%mínimo-35\%máximo; pero al realizar la comparación entre los resultados obtenidos está por debajo de lo establecido, esta variación de humedad del pan se debe al tipo de harina que se utilizó en la elaboración de dicho producto (Pilataxi, 2013).

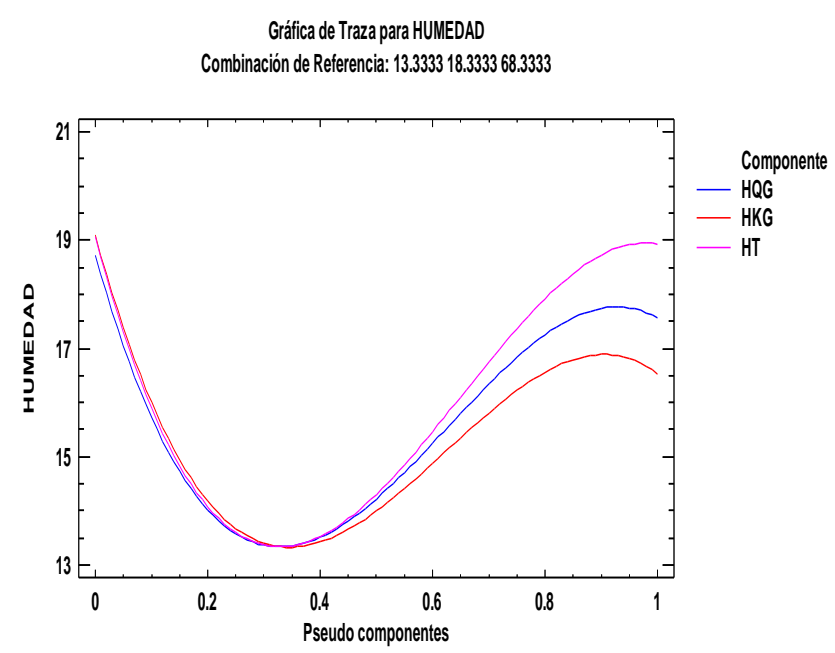

Figura 7. Gráfica de trazas para la humedad del pan.

Proteína del pan: En la figura 8 se muestra el valor máximo del \% proteico del pan que alcanza con la HKG mientras que el valor mínimo con HQG.

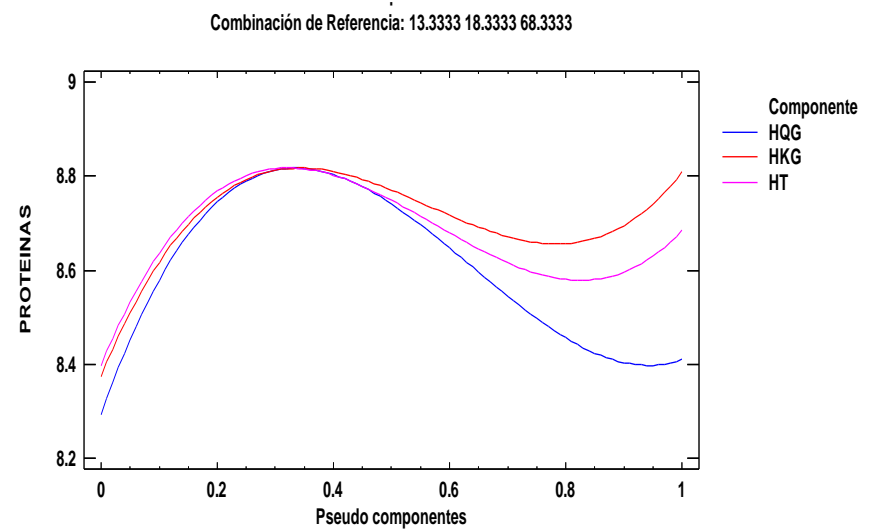

Figura 8. Gráfica de trazas para la proteína del pan.

Por otra parte, Vásquez, et al. (2016) y Arone (2015) mencionan que al realizar la comparación entre un pan normal y los panes con $\mathrm{HQ}$ a diferentes \% da a conocer que el \% de proteínas en el pan tiende a incrementarse a medida que aumenta el \% de sustitución. 
Grasa del pan: En la figura 9 se muestra el porcentaje de grasa del pan siendo HQG el de mayor efecto seguido de HT por último con menor efecto la HKG.

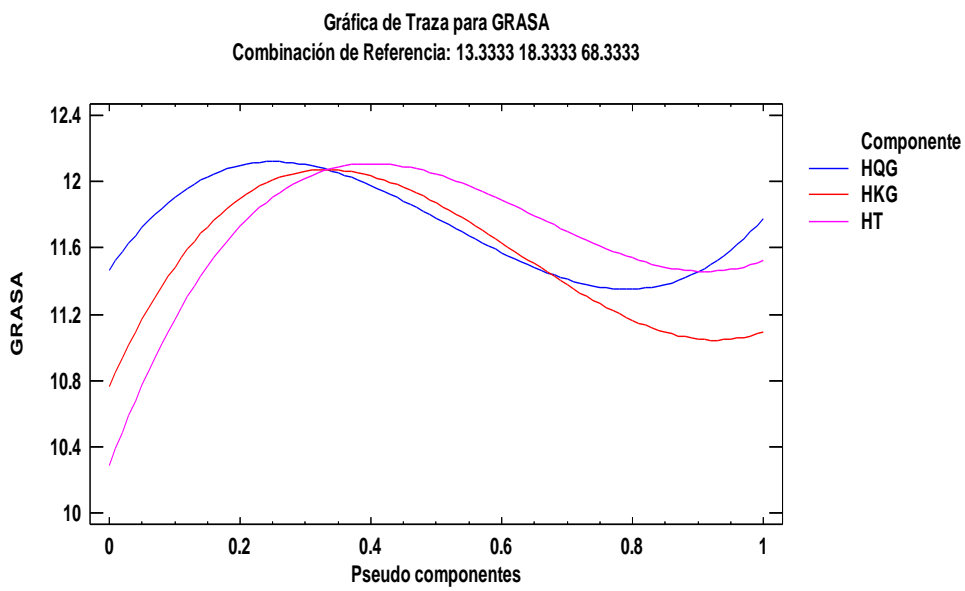

Figura 9. Gráfica de trazas para la grasa del pan.

Para Vásquez, et al. (2016) menciona que él \% de grasa en el pan tiende a incrementarse a medida que aumenta el \% de sustitución; en la elaboración del pan generalmente se usa entre 11 y $15 \%$ de grasa dependiendo al tipo de pan, sin embargo, en la investigación solo se usó $10 \%$ de grasa.

Ceniza del pan: En la figura 10 se muestra el efecto en el porcentaje de ceniza del pan siendo el mayor la HQG, en segundo lugar, la HKG y con menor efecto la HT.

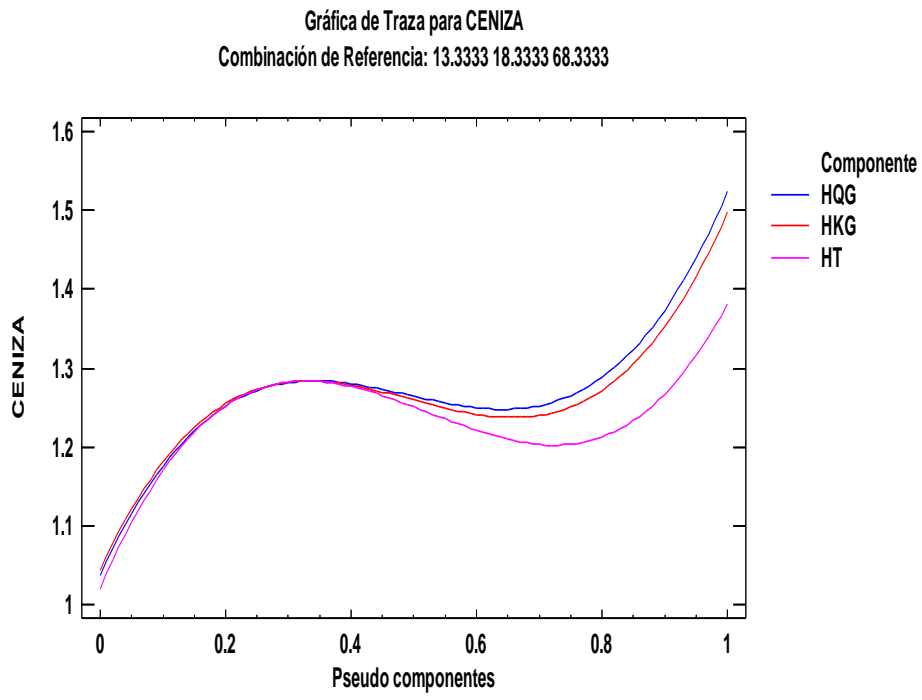

Figura 10. Gráfica de trazas para la ceniza del pan. 
Para Bodroza (como cito Pilataxi, 2013) menciona que el incremento del \% de ceniza en el pan es un efecto positivo ya que la cantidad de cenizas indica el contenido de minerales, entre ellos se encuentran: el calcio, fosforo, magnesio, potasio, sodio, cobre, manganeso, zinc.

Fibra del pan: En la figura 11 se aprecia el valor máximo del \% de fibra del pan se alcanza con la HKG, mientras que el valor mínimo se logra con HT.

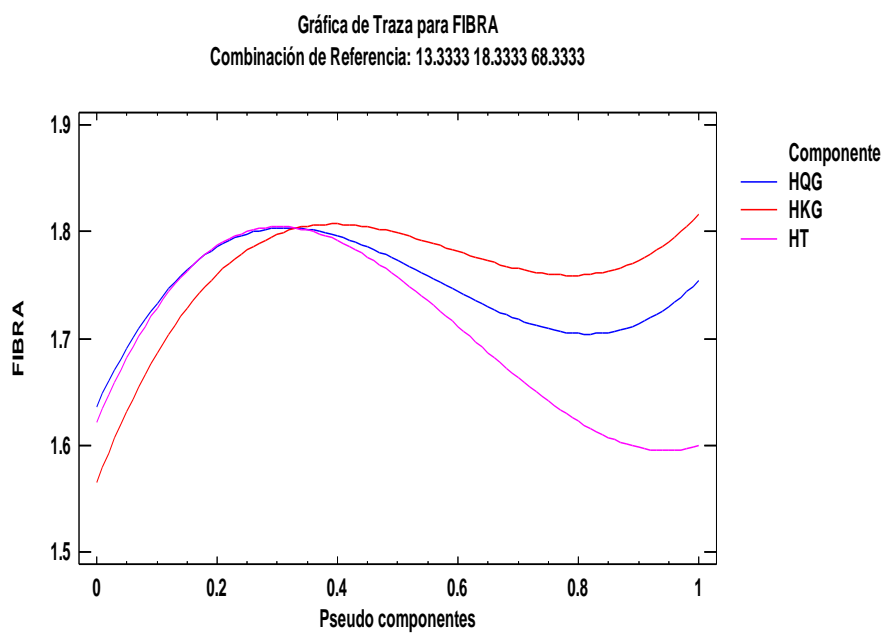

Figura 11. Gráfica de trazas para la fibra del pan.

Según Salas (2017), da a conocer que, con la formulación que contenga mayor cantidad de harina de un grano germinado, se conseguirá un aumento del contenido de fibra cruda.

Carbohidrato del pan: El valor óptimo para él \% de carbohidrato es 62.52 con una combinación óptima de $\mathrm{HQG}=13.5 \%$, $\mathrm{HKG}=18.5 \%$ y $\mathrm{HT}=67.97 \%$.

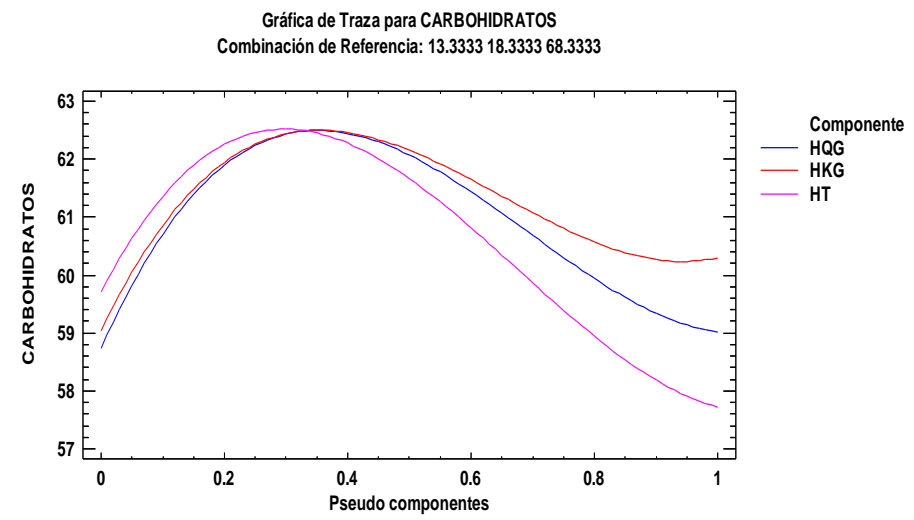

Figura 12. Gráfica de trazas para el carbohidrato del pan. 
Vásquez, et al. (2016) y Salas (2017), dan a conocer que el \% de carbohidratos en el pan tiende a disminuir a medida que aumenta el \% de sustitución. Por otra parte, gracias a la germinación de la quinua y kiwicha se logra convertir los hidratos de carbono más complejos como el almidón en azucares más simples (Cruz, 2017).

\section{Conclusiones}

Se logró elaborar pan tipo bollo con sustitución parcial de harina de trigo por harina de quinua germinada y harina de kiwicha germinada y el total de harina sumaba el $100 \%$ del cual solo $35 \%$ estaba conformada por la HQG y HKG el $65 \%$ es HT.

Respecto a las características químicas del pan, el nivel de sustitución si tuvo efecto significativo en el \% de humedad, \% ceniza y \% de carbohidratos, sin embargo, no tuvo un efecto significativo en el \% de proteínas, \% de grasa y \% de fibra. El tratamiento que presenta mejores características fisicoquímicas es T1 cuya distribución porcentual de harinas fueron las siguientes $\mathrm{HQG}=10 \%, \mathrm{HKG}=25 \%$ y $\mathrm{HT}=65 \%$.

Se determinó las características físicas del pan entre ellas el peso promedio del pan fue de $60.22 \mathrm{~g}$, con relación a la textura presento variaciones significativas, a medida que disminuye el \% de harina de quinua germinada (HQG) y aumente el \% de harina de kiwicha (HKG), el valor de la textura del pan se incrementa, y el volumen especifico no presentó una diferencia significativa entre los tres tratamientos ya que del $100 \%$ el $35 \%$ es sustitución con HQG y HKG, finalmente la porosidad del pan no tiene diferencia significativa porque el porcentaje de variación en cada tratamiento fue del $35 \%$ y el $65 \%$ estaba conformado por HT obteniendo un $67.48 \%$ de porosidad promedio del pan.

\section{Referencias}


Arone, H. (2015) Evaluación de las propiedades físicas, químicas y organolépticas del pan tipo molde enriquecido con harina de quinua (Chenopodium quinoa Willd) y chía (Salvia hispánica L.). (Tesis de pregrado).

Botero, A. (1a ed.). (2011) Los germinados como alimento excepcional y medicina natural. Bogotá, Colombia.

Callejo, M. (2002) Industrias de Cereales y Derivados. Madrid: Editorial AMV-MundiPrensa.

Cauvain, S. y Young, L. (1998) Fabricación del pan. Zaragoza, España: Editorial Acribia.

Cruz, V. (2017) Aplicaciones de la semilla germinada de quinua (Chenopodium quinoa Willd) en la mejora Nutricional de los preparados alimentarios. (Tesis de pregrado).

Edel, L. y Rosell, C. (1a ed.). (2007) De tales harinas, tales panes: granos, harinas y productos de panificación en lberoamérica. Córdoba, Argentina.

FAO/OMS/ONU. (1985). Necesidades de energía y de proteínas.

Pilataxi, M. (2013) Elaboración y evaluación nutritiva y nutracéutica de pan con harina de amaranto (Amaranthus caudatus). (Tesis de pregrado).

Sahin, S. y Gülüm Sumnu, S. (2009) Propiedades físicas de los alimentos. Zaragoza: Editorial Acriba S. A.

Salas, M. (2017) Desarrollo de un pan dulce enriquecido con harina de germinado de trigo (Triticum aestivum AN-91-98). (Tesis de pregrado)

Tejero, F. (2 Vols.). (1992) Panadería Española. Barcelona: Editorial Montagud.

Vásquez, F.; Verdú, S.; Islas, A. R.; Barat, J. M. y Grau, R. (2016) Efecto de la sustitución de harina de trigo con harina de quinoa (Chenopodium quinoa) sobre las propiedades reológicas de la masa y texturales del pan. Revista Iberoamericana de Tecnología Postcosecha, vol. 17, núm. 2,2016, pp. 307-317, Hermosillo, México.

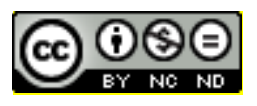

\section{Caracterización Fisicoquímica de Pan con Sustitución Parcial de Harina de Trigo} por Harina de Quinua (chenopodium quinoa willd) y Kiwicha (amaranthus caudatus

I.) Germinadas. (Dr. Jorge Wilmer Elías - Silupu) Por Revista Sendas se encuentra bajo una Licencia Creative Commons-No Comercial-Sin Derivadas 3.0 Uported 